\title{
Archimedean-like tiling on decagonal quasicrystalline surfaces
}

\author{
Jules Mikhael ${ }^{1}$, Johannes Roth ${ }^{2}$, Laurent Helden ${ }^{1} \&$ Clemens Bechinger ${ }^{1,3}$
}

\begin{abstract}
Monolayers on crystalline surfaces often form complex structures with physical and chemical properties that differ strongly from those of their bulk phases ${ }^{1}$. Such hetero-epitactic overlayers are currently used in nanotechnology and understanding their growth mechanism is important for the development of new materials and devices. In comparison with crystals, quasicrystalline surfaces exhibit much larger structural and chemical complexity leading, for example, to unusual frictional ${ }^{2}$, catalytical ${ }^{3}$ or optical properties $^{4,5}$. Deposition of thin films on such substrates can lead to structures that may have typical quasicrystalline properties. Recent experiments have indeed showed 5-fold symmetries in the diffraction pattern of metallic layers adsorbed on quasicrystals ${ }^{6,7}$. Here we report a real-space investigation of the phase behaviour of a colloidal monolayer interacting with a quasicrystalline decagonal substrate created by interfering five laser beams. We find a pseudomorphic phase that shows both crystalline and quasicrystalline structural properties. It can be described by an archimedean-like tiling ${ }^{8,9}$ consisting of alternating rows of square and triangular tiles. The calculated diffraction pattern of this phase is in agreement with recent observations of copper adsorbed on icosahedral $\mathrm{Al}_{70} \mathrm{Pd}_{21} \mathrm{Mn}_{9}$ surfaces $^{10}$. In addition to establishing a link between archimedean tilings and quasicrystals, our experiments allow us to investigate in real space how single-element monolayers can form commensurate structures on quasicrystalline surfaces.
\end{abstract}

Quasicrystals are unusual materials: they are aperiodic but retain true long-range order ${ }^{11}$. Although quasicrystalline structures have been theoretically also predicted in systems with a single type of

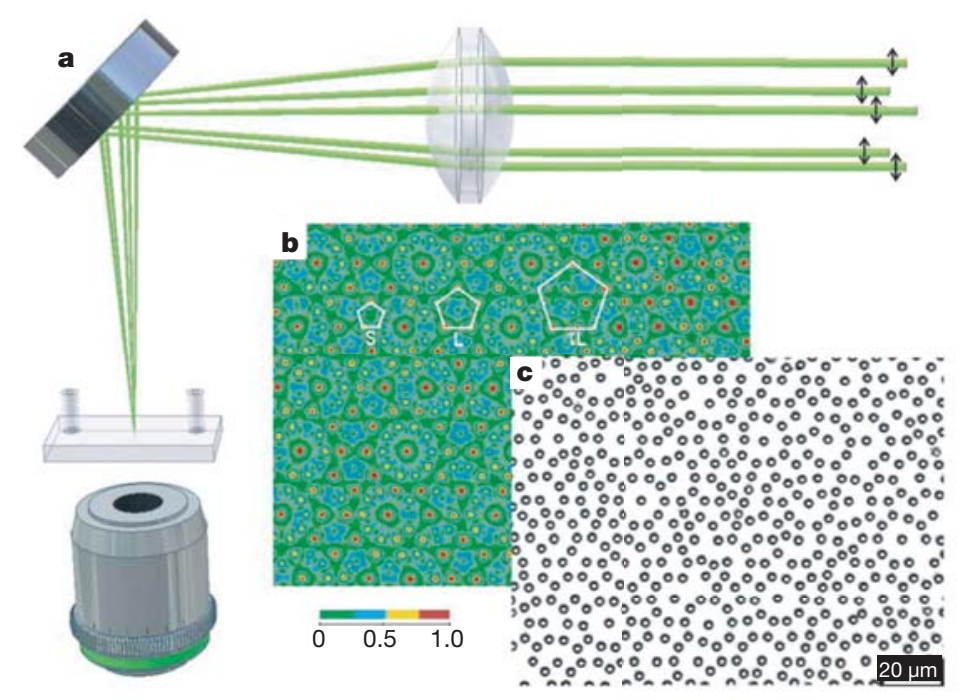

particle $^{12,13}$, experimentally their spontaneous formation has been observed only in binary, ternary or even more complex alloys ${ }^{14}$. Accordingly, their surfaces exhibit a high degree of structural and chemical complexity and show unexpected mechanical, electrical and optical properties ${ }^{15}$. To understand the origin of those characteristics it is useful to disentangle structural and chemical aspects; this can be achieved by growing single-element monolayers to quasicrystalline surfaces ${ }^{16,17}$. Apart from adding to our understanding of how quasicrystalline properties can be transferred to such monolayers ${ }^{18}$, this approach might permit the fabrication of materials with previously unobserved properties. Heteroepictatic growth experiments on decagonal and icosahedral surfaces did indeed show the formation of $\mathrm{Bi}$ and $\mathrm{Sb}$ monolayers with a high degree of quasicrystalline order as determined by low-energy electron diffraction and elastic heliumatom scattering experiments ${ }^{6,18}$. In comparison with reciprocal space studies, it was only recently that scanning tunnelling microscopy permitted an atomic resolution of the adsorbate morphology ${ }^{7}$. Even then, however, it was difficult to relate the structure of the adsorbate to that of the underlying substrate.

Here we report an experimental study of the phase behaviour of a two-dimensional colloidal monolayer in the presence of a quasicrystalline substrate potential. The quasicrystalline substrate potential is created by the interference of five laser beams in the sample plane (Fig. 1a, b). As a result of optical gradient forces ${ }^{19}$, this light pattern acts as a decagonal quasicrystalline surface for the colloidal particles $^{20,21}$. Figure $1 \mathrm{~b}$ shows the light intensity distribution in the sample plane, which displays maxima arranged in pentagons of different
Figure 1 | Experimental realization of colloidal quasicrystals. a, Five linearly polarized (polarization as indicated by arrows) parallel laser beams forming a regular pentagon are focused with an achromatic lens into a thin sample cell. b, Experimentally determined intensity distribution of the interference pattern, which acts as a substrate potential for the colloids. The pattern displays a decagonal symmetry and the predominating motifs are pentagons (indicated in white) with sides of different lengths related by the golden ratio $\tau=L / S$. Here $S=5.64 \mu \mathrm{m}$ and $L=9.13 \mu \mathrm{m}$. The colour coding of the intensity field ranges from green to red and reflects the variation in potential well depth. c, Configuration of colloidal particles at a density of $\Phi=0.0264 \mu^{-2}$ exposed to a decagonal substrate interference pattern. 
sizes whose side lengths and heights are related by the golden ratio $\tau=(1+\sqrt{5}) / 2=1.618 \ldots$. The substrate strength can be continuously adjusted by the laser intensity $I_{0}$ (ref. 22). As a colloidal suspension we used highly charged polystyrene spheres of radius $R=1.45 \mu \mathrm{m}$ suspended in water in a silica cuvette with a height of $200 \mu \mathrm{m}$. Particles at distance $r$ interact through a repulsive screened electrostatic-pair potential $u(r) \propto \frac{1}{r} \exp (-\kappa r)$ whose range depends on the Debye screening length $\kappa^{-1}$ given by the ion concentration of the suspension ${ }^{23}$. Particle positions are observed in real space with digital video microscopy, which allows us to determine their positions relative to the substrate potential with a precision of about $50 \mathrm{~nm}$ (Fig. 1c). From the particle coordinates we obtain the particle density distribution $\rho(x, y)$ and the two-dimensional structure factor $S\left(q_{x}, q_{y}\right)$, the latter being equivalent to the diffraction pattern. In addition we calculated the projection of the structure factor on the $q_{x}$ axis, $\bar{S}\left(q_{x}\right)$, which corresponds to a slice in the real-space structure along the projection direction ${ }^{14}$.

Figure 2 a-d shows how $\rho(x, y)$ and $S\left(q_{x}, q_{y}\right)$ of a colloidal monolayer with a particle density $\Phi=0.0465 \mu \mathrm{m}^{-2}$ and $\kappa^{-1} \approx 160 \mathrm{~nm}$ changes when the substrate strength of the decagonal substrate is increased. In absence of a substrate potential $\left(I_{0}=0\right)$ the system crystallizes as shown in Fig. 2a, b. The observed structure can be described by a triangular lattice (green tiles) whose vertices are defined by the maxima of $\rho(x, y)$. Apart from some defects, each vertex is surrounded by six triangular tiles forming a $\left(3^{6}\right)$-vertex type. The crystalline structure is confirmed by the diffraction pattern, which has 6 -fold coordinated spots leading to periodically spaced peaks in the projected diffraction pattern $\bar{S}\left(q_{x}\right)$ (Fig. 2b). The intensity of the peaks decreases with increasing diffraction order, reflecting the thermal motion of particles $^{24}$. In the presence of a quasicrystalline light field, the particles also interact with the corresponding surface potential, and the equilibrium structure will change. To establish equilibrium conditions, the laser intensity $I_{0}$ was increased at a rate much smaller than the typical relaxation time of the colloidal system. At low $I_{0}$, the electrostatic colloidal repulsion dominates over the colloid-substrate interaction and the crystalline structure remains mainly intact. In agreement with numerical simulations of weakly adsorbed atomic systems, we observe the alignment of crystalline domains along the 5 -fold directions of the quasicrystalline substrate $e^{25}$.

For $I_{0}>1.3 \mu \mathrm{W} \mu \mathrm{m}^{-2}$, however, we observe a structure that shows neither a triangular nor a decagonal symmetry. As an example we show the situation for $I_{0}=2 \mu \mathrm{W} \mu \mathrm{m}^{-2}$ (Fig. 2c, d). In contrast with a triangular structure, in which the nearest-neighbour distance distribution has a single peak, here it is bimodal (data not shown). The structure is well characterized by a tiling composed of squares (blue) and triangles (green), with the vertex-vertex bonds being selected by their length and angle (for details see Methods). The tiling structure has rows of triangles and squares mainly aligned in one direction with some intrusions at an angle of $72^{\circ}$. The direction of the rows varied between different experiments but always corresponded to one of the five orientations given by the substrate potential. The peaks in $S\left(q_{x}, q_{y}\right)$ are periodically spaced along the $q_{x}$ direction (as clearly seen in the projection $\bar{S}\left(q_{x}\right)$ ), whereas in the $q_{y}$ direction their distance is close to the golden ratio $\tau$ (see vertical spacing of white arrows). This is a clear signature of quasicrystalline order along the $y$ direction. Obviously, the competition between the colloid-colloid repulsion and their interaction with the quasicrystalline substrate leads to a phase that has both periodic and quasicrystalline structural properties.
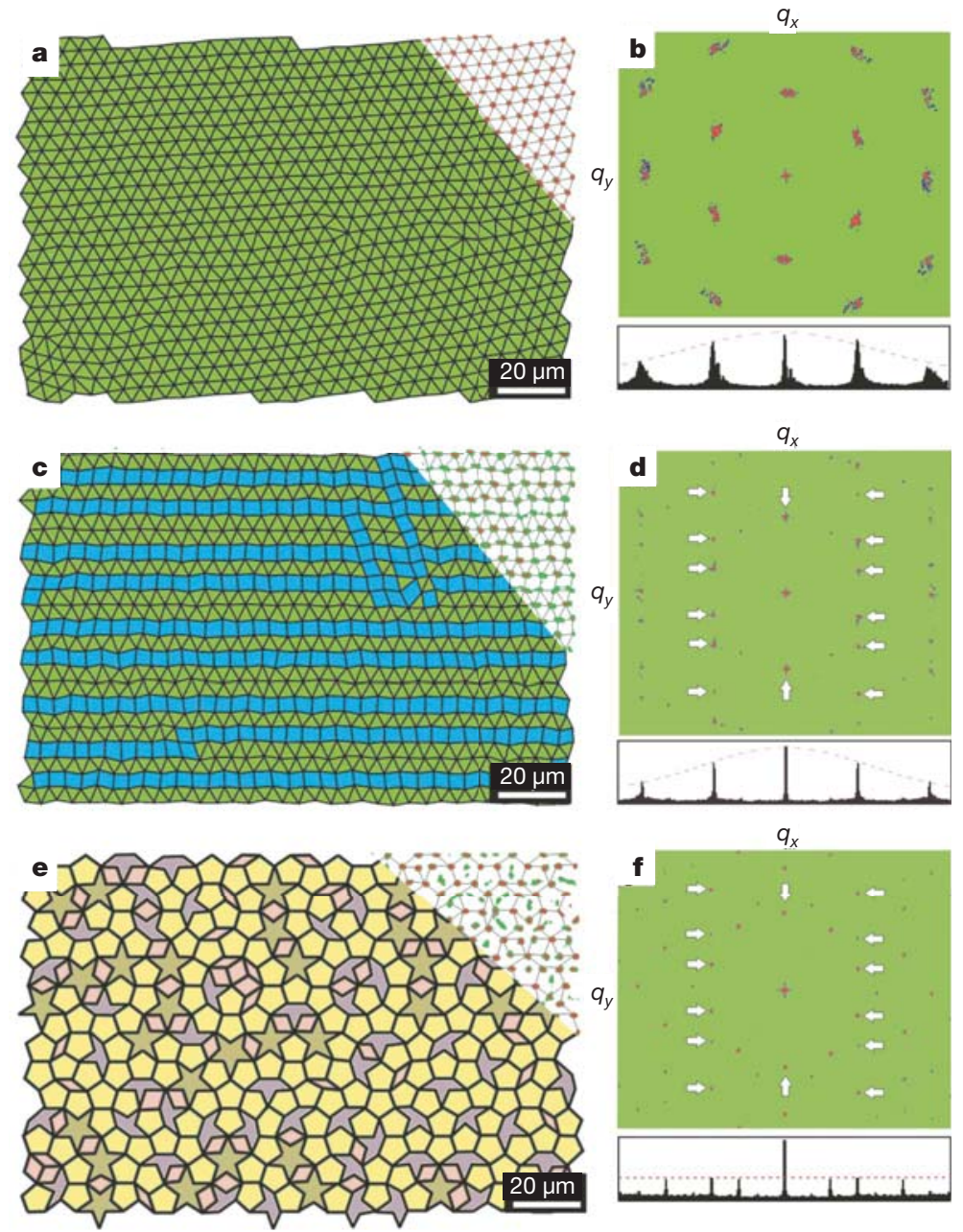

Figure 2 | Real and reciprocal space structure of the adsorbate. a, b, Crystalline phase $\left(I_{0}=0\right.$, $\left.\kappa^{-1} \approx 160 \mathrm{~nm}\right)$. a, Particle density distribution (shown in the upper right corner) and tiling with triangles. Increasing particle density is labelled from white through green to red. $\mathbf{b}$, Diffraction pattern and projection $\bar{S}\left(q_{x}\right)$. c, d, Intermediate phase $\left(I_{0}=2 \mu \mathrm{W} \mu \mathrm{m}^{-2}, \kappa^{-1} \approx 160 \mathrm{~nm}\right)$. c, Particle density and tiling of colloidal monolayer subjected to a decagonal light lattice. The tiling consists of rows containing triangles (green) and squares (blue). d, $S\left(q_{x}, q_{y}\right)$ and $\bar{S}\left(q_{x}\right)$. The arrows indicate diffraction peaks also found in the quasicrystalline phase in f. e, f, Quasicrystalline phase $\left(I_{0}=2 \mu \mathrm{W} \mu \mathrm{m}^{-2}\right.$, $\left.\kappa^{-1} \approx 10 \mathrm{~nm}\right)$. e, Particle density and tiling composed of pentagons (yellow), rhombuses (pink), crowns (violet) and pentagonal stars (brown). f, $S\left(q_{x}, q_{y}\right)$ with 10 -fold symmetry and $\bar{S}\left(q_{x}\right)$ with peak spacing given by $\tau$. 
Before analysing this new intermediate phase in more detail, we discuss the observations when the colloid-substrate interaction dominates. This is achieved by increasing the ionic strength of the suspension, which greatly reduces the repulsion between the colloids. For $I_{0}=2 \mu \mathrm{W} \mu \mathrm{m}^{-2}$ and Debye screening lengths $\kappa^{-1} \leq 95 \pm 15 \mathrm{~nm}$, the particle density distribution changes greatly in comparison with Fig. $2 \mathrm{c}$, and the maxima $\left(\rho \geq 0.7 \rho_{\max }\right)$ now follow a tiling consisting of prototiles shaped like rhombuses, pentagons, crowns and stars (Fig. 2e). Such tiles are known from the P1 Penrose tiling and have also been applied successfully to the 5-fold surface of AlPdMn (ref. 26). The corresponding diffraction pattern shows perfect quasicrystalline order and thus mimics the geometry of the underlying light potential (Fig. 2f). In the $q_{y}$ direction the diffraction spots (arrows) coincide with those of the intermediate phase but, unlike in Fig. 2d, here $S\left(q_{x}, q_{y}\right)$ has a 10 -fold rotational symmetry. As expected, $\bar{S}\left(q_{x}\right)$ consists of peaks whose distances are not equal but are given by $\tau$. In contrast to Fig. 2 d, the intensity of the peaks of $\bar{S}\left(q_{x}\right)$ is constant. This is consistent with the fact, that as a result of the strong substrate interactions the thermal motion of the particles becomes reduced ${ }^{24}$.

The structure of the intermediate phase is remarkably similar to one of the 11 archimedean tilings ${ }^{8}$ first introduced by Kepler in 1619 . Currently, there is renewed interest in archimedean tilings as candidates for photonic crystals' ${ }^{9}$. In contrast to the five two-dimensional Bravais lattices each described by identical tiles (being the corresponding unit cell), archimedean tilings may be composed of more than one, but regular, tile. Those tiles are arranged in such a way that only one vertex type exists. Figure 3a shows an example of an archimedean tiling consisting of alternating rows of triangular and square tiles. Because each vertex is surrounded by three triangles and two squares, this leads to a $\left(3^{3} .4^{2}\right)$-vertex type. Although the structure is strictly periodic, it has marked similarities with quasicrystals. First, every vertex has five nearest neighbours at equal distances that form an irregular pentagon. Second, the structure of the archimedean tiling is equivalent to an oblique lattice (red lines) with a two-atomic basis. The oblique angle $\gamma=75^{\circ}$ is close to the value of $72^{\circ}$ on decagonal substrates. Accordingly, when superimposing ideal pentagons (white lines) on the archimedean tiling, their vertices (and the centre of the bigger one) agree almost perfectly with the vertex positions. The height ratio of these pentagons equals the golden ratio $\tau$.

To understand how the observed intermediate phase forms on the quasicrystalline substrate, we plotted the contours of the tiles taken from Fig. 2c (black lines) on top of the decagonal intensity distribution of the laser field (Fig. 3b). The deepest potential wells of the substrate coincide with vertices and thus show quasicrystalline order. For our particle density, this applies to about half of all vertex positions. The other vertices are located at sites with weak or vanishing substrate interactions, and their configuration is dominated by electrostatic particle repulsion. They therefore assemble in such a way that their nearest-neighbour distance is fairly uniform. As a result, vertices partly adopt a 5-fold rotational symmetry but simultaneously seek to achieve equal nearest-neighbour distances. Both aspects are ideally supported by the archimedean tiling.

Because archimedean tilings are strictly periodic, they can be only locally commensurate with quasicrystalline substrates; disruptions at larger length scales must occur. Indeed, the tiling of the intermediate phase in Fig. 2c shows additional interstitial rows of triangles (that is, double triangular rows). As a result, two vertex types, namely $\left(3^{3} \cdot 4^{2}\right)$ and $\left(3^{6}\right)$, arise. Such structures are referred to as archimedean-like tilings ${ }^{9}$. One would expect that the spacing of those interstitial rows corresponds to a Fibonacci sequence, taking into account the longrange quasicrystalline order along one direction. This is consistent with the structure observed in Fig. 2c. The origin of the two characteristic length scales of such a Fibonacci chain is due to the height of small and big pentagons as shown in Fig. 3b. Whenever two pentagons adjoin, the periodic sequence of triangular and squared rows becomes disrupted and an additional row of triangular tiles is inserted.

We also investigated whether the intermediate phase is stable with other parameters. Figure $3 \mathrm{c}$ shows the result when a colloidal layer with density $\Phi=0.0307 \mu \mathrm{m}^{-2}$ is exposed to a decagonal substrate potential whose characteristic length scales are decreased to about $70 \%$ compared with Fig. $3 \mathrm{~b}$. In contrast to the above, here the number of deep potential wells provided by the substrate is larger than the number of vertices. Nevertheless, the system's structure is again well described by an archimedean-like tiling and even though the magnitude of particle fluctuations relative to the substrate is stronger than in Fig. 3b, the diffraction pattern (Fig. 3d) agrees well with that in Fig. 2 d. This is more clearly seen in the projection of $S\left(q_{x}, q_{y}\right)$, which shows again equidistant peaks with decreasing intensity and suggests that the intermediate phase forms for a wider range of parameters.

Because the phase behaviour of colloidal monolayers on surfaces is similar to that of atomic systems ${ }^{27}$, we expect that structures comparable to those in Fig. 3 should occur in atomic adsorbates on quasicrystalline surfaces. Recent experiments with thin copper films
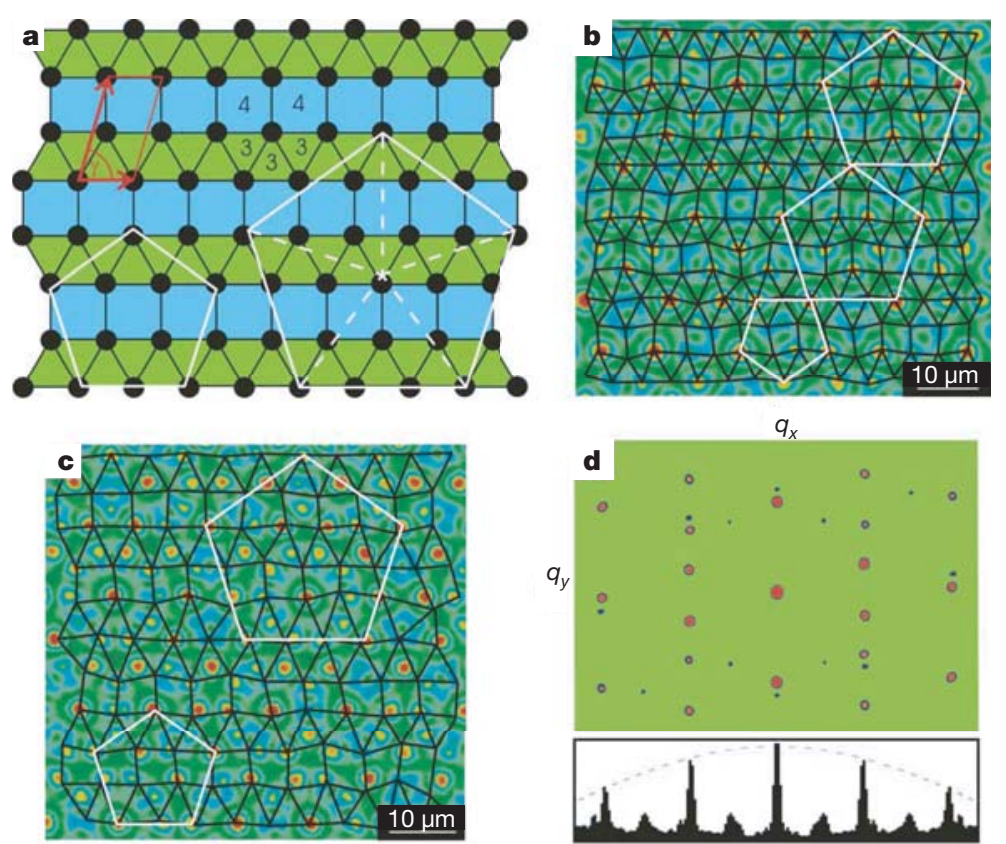

Figure 3 | Substrate-adsorbate correlations. a, Ideal archimedean tiling with $\left(3^{3} .4^{2}\right)$ vertex type. The vertices and centres of the two pentagons (white) fit the lattice sites almost perfectly. The structure can be also represented by an oblique lattice with two atoms per unit cell (red). b, Tiling of the intermediate phase superimposed on the laser intensity distribution of the decagonal interference pattern $(S=5.65 \mu \mathrm{m}, L=9.14 \mu \mathrm{m})$. Particles are partly located at deep minima in substrate potential, thus showing quasicrystalline order as indicated by the pentagons (white). Other colloids are located at interstitial sites with weak substrate interactions. c, As in b except for $\Phi=0.0307 \mu \mathrm{m}^{-2}$ and $S=4.10 \mu \mathrm{m}, L=6.63 \mu \mathrm{m}$. d, Structure factor and $\bar{S}\left(q_{x}\right)$ of c, which is similar to that in Fig. 2d. 
deposited on the 5-fold surface of icosahedral AlPdMn quasicrystals reveal that above a few monolayers the copper atoms are arranged in rows spaced in a Fibonacci sequence ${ }^{10,28}$. The atomic positions relative to the substrate have not yet been identified. However, although the atomic pair interactions in these experiments are more complex than in colloidal systems, the diffraction pattern of the copper film is almost identical to that of our intermediate phase (see Supplementary Fig. 1). This close resemblance suggests that the intermediate phase does not necessarily require complex substrate interactions but is driven by geometrical considerations and thus might also be observed for other adsorbate/quasicrystal combinations.

Our experiments show that colloidal systems on decagonal light patterns allow us to understand the equilibrium structure of monolayers on quasicrystalline surfaces. This approach can be also extended to investigate dynamical processes on quasicrystalline surfaces. By introducing phase shifts between the interfering laser beams, phason ${ }^{29}$ or phonon modes can be induced in the substrate. These elementary excitations are important for the three-dimensional growth of quasicrystals and it will be interesting to study how such substrate excitations modify the behaviour of adsorbed thin films.

\section{METHODS SUMMARY}

The particle density $\Phi$ is adjusted by an optical fence created by an additional laser beam $(\lambda=488 \mathrm{~nm})$, which was scanned around the central region of the sample to create a boundary box ${ }^{30}$. Its size can be continuously adjusted by a pair of computer-controlled scanned mirrors (Scanlab AG). With this technique, the particle density can be adjusted within a precision of about $1 \%$. An additional laser beam $(\lambda=514 \mathrm{~nm})$, which is vertically incident on the sample cell from above, pushes the colloids towards the negatively charged substrate and reduces vertical particle fluctuations to less than $5 \%$ of the particle radius. The system can therefore be considered to be two-dimensional.

As colloidal particles we used polystyrene particles with a radius $R$ of $1.45 \mu \mathrm{m}$, a polydispersity of $4 \%$ and a negative surface charge density of $9.8 \mu \mathrm{C} \mathrm{cm}^{-2}$ (batch no. 1212; IDC). To reduce the ionic strength, we deionized the sample cell with a deionization circuit containing a vessel of ion-exchange resin, an electrical conductivity probe to measure the ionic concentration, and a peristaltic pump. Afterwards the suspension was inserted into the cell, which was then sealed. The Debye screening length was determined from the measured pair correlation function in the absence of the quasicrystalline lattice ${ }^{30}$.

For the identification of phases we performed a Delaunay triangulation of the vertices as obtained by the maxima of $\rho(x, y)$ to identify next-neighbour bonds. The bond length distribution depends sensitively on the different phases, as follows: first, crystal, a monomodal distribution with a peak located at the mean particle distance; second, intermediate, a bimodal distribution with the ratio of the peak positions close to $\sqrt{2}$ and none of the peaks located at $\mathrm{S}$ and $\mathrm{L}$ (when the longer bonds are removed from the triangulation, one obtains square and triangular tiles as shown in Fig. 2c); and third, quasicrystalline, a bimodal distribution with peaks at $\mathrm{S}$ and $\mathrm{L}$.

1. Barth, J. V., Costantini, G. \& Kern, K. Engineering atomic and molecular nanostructures at surfaces. Nature 437, 671-679 (2005).

2. Park, J. Y. et al. High frictional anisotropy of periodic and aperiodic directions on a quasicrystal surface. Science 309, 1354-1356 (2005).

3. Tsai, A. P. \& Yoshimura, M. Highly active quasicrystalline Al-Cu-Fe catalyst for steam reforming of methanol. Appl. Catal. A 214, 237-241 (2001).

4. Zoorob, M. E. Charlton, M. D. B. Parker, G. J., Baumberg, J. J. \& Netti, M. C. Complete photonic bandgaps in 12-fold symmetric quasicrystals. Nature 404,740-743 (2000).

5. Matsui, T., Agrawal, A., Nahata, A. \& Vardeny, Z. V. Transmission resonances through aperiodic arrays of subwavelength apertures. Nature 446, 517-521 (2007).
6. Franke, K. J. et al. Quasicrystalline epitaxial single element monolayers on icosahedral Al-Pd-Mn and decagonal Al-Ni-Co quasicrystal surfaces. Phys. Rev. Lett. 89, 156104 (2002)

7. Sharma, H. R., Shimoda, M., Ross, A. R., Lograsso, T. A. \& Tsai, A. P. Real-space observation of quasicrystalline $\mathrm{Sn}$ monolayer formed on the fivefold surface of icosahedral Al-Cu-Fe quasicrystal. Phys. Rev. B 72, 045428 (2005).

8. Pearce, P. Structure in Nature is a Strategy for Design (MIT Press, Cambridge, MA, 1978)

9. David, S., Chelnokov, A. \& Lourtioz, J. M. Isotropic photonic structures: Archimedean-like tilings and quasi-crystals. IEEE J. Quantum Electron. 37, 1427-1434 (2001).

10. Ledieu, J. et al. Copper adsorption on the fivefold $\mathrm{Al}_{70} \mathrm{Pd}_{21} \mathrm{Mn}_{9}$ quasicrystal surface. Phys. Rev. B 72, 035420 (2005).

11. Shechtman, D., Blech, I., Gratias, D. \& Cahn, J. W. Metallic phase with long-range orientational order and no translational symmetry. Phys. Rev. Lett. 53, 1951-1953 (1984).

12. Engel, M. \& Trebin, H.-R. Self-assembly of monoatomic complex crystals and quasicrystals with a double-well interaction potential. Phys. Rev. Lett. 98, 225505 (2007)

13. Keys, A. S. \& Glotzer, S. C. How do quasicrystals grow? Phys. Rev. Lett. 99, 235503 (2007)

14. Janot, C. Quasicrystals-A Primer (Oxford Univ. Press, New York, 1994)

15. Dubois, J. M. Quasicrystals. J. Phys. Condens. Matter 13, 7753-7762 (2001)

16. Fournee, $\mathrm{V}$. et al. Nucleation and growth of $\mathrm{Ag}$ films on a quasicrystalline AIPdMn surface. Phys. Rev. B 67, 033406 (2003)

17. Curtarolo, S., Setyawan, W., Ferralis, N., Diehl, R. D. \& Cole, M. W. Evolution of topological order in Xe films on a quasicrystal surface. Phys. Rev. Lett. 95, 136104 (2005).

18. Sharma, H. R., Shimoda, M. \& Tsai, A. P. Quasicrystal surfaces: structure and growth of atomic overlayers. Adv. Phys. 56, 403-464 (2007).

19. Ashkin, A. Optical trapping and manipulation of neutral particles using lasers. Proc. Natl Acad. Sci. USA 94, 4853-4860 (1997).

20. Burns, M. M., Fournier, J. M. \& Golovchenko, J. A. Optical matter-crystallization and binding in intense optical fields. Science 249, 749-754 (1990).

21. Roichman, Y. \& Grier, D. G. Holographic assembly of quasicrystalline photonic heterostructures. Opt. Express 13, 5434-5439 (2005)

22. Bechinger, C., Brunner, M. \& Leiderer, P. Phase behavior of two-dimensional colloidal systems in the presence of periodic light fields. Phys. Rev. Lett. 86, 930-933 (2001).

23. Yethiraj, A. Tunable colloids: control of colloidal phase transitions with tunable interactions. Soft Matter 3, 1099-1115 (2007).

24. Guinier, A. X-ray Diffraction-In Crystals, Imperfect Crystals and Amorphous Bodies (Dover, New York, 1994).

25. Bilki, B., Erbudak, M., Mungan, M. \& Weisskopf, Y. Structure formation of a layer of adatoms on a quasicrystalline substrate: Molecular dynamics study. Phys. Rev. B 75, 045437 (2007)

26. Ledieu, J. et al. Tiling of the fivefold surface of $\mathrm{Al}_{70} \mathrm{Pd}_{21} \mathrm{Mn}_{9}$. Surf. Sci. 492, L729-L734 (2001)

27. Yethiraj, A. Tunable colloids: control of colloidal phase transitions with tunable interactions. Soft Matter 3, 1099-1115 (2007).

28. Ledieu, J. et al. Pseudomorphic growth of a single element quasiperiodic ultrathin film on a quasicrystal substrate. Phys. Rev. Lett. 92, 135507 (2004).

29. Freedman, B., Lifshitz, R., Fleischer, J. W. \& Segev, M. Phason dynamics in nonlinear photonic quasicrystals. Nature Mater. 6, 776-781 (2007)

30. Brunner, M., Bechinger, C., Strepp, W., Lobaskin, V. \& v Grünberg, H. H. Densitydependent pair-interactions in 2D colloidal suspensions. Europhys. Lett. 58, 926-932 (2002) 